\title{
Pathway-related modules involved in the application of sevoflurane or propofol in off-pump coronary artery bypass graft surgery
}

\author{
XIANGMEI BU ${ }^{1,2}$, BO WANG $^{3}$, YAOQI WANG ${ }^{2}$, ZHIGANG WANG $^{1}$, \\ CHUNZHI GONG ${ }^{2}$, FENG QI ${ }^{1}$ and CAIXIA ZHANG ${ }^{4}$ \\ ${ }^{1}$ Department of Anesthesiology, Qilu Hospital of Shandong University, Jinan, Shandong 250012; \\ Departments of ${ }^{2}$ Anesthesiology, ${ }^{3}$ Neurosurgery, and ${ }^{4}$ Gerontonogy \& Health Care General \\ Medicine, Binzhou Medical University Hospital, Binzhou, Shandong 256603, P.R. China
}

Received February 15, 2016; Accepted February 17, 2017

DOI: $10.3892 / \mathrm{etm} .2017 .4504$

\begin{abstract}
Off-pump coronary artery bypass graft (CABG) surgery has recently emerged as a means to avoid the sequelae of extracorporeal circulation, including the whole-body inflammatory response, coagulation disorders and multiple organ dysfunction. At present, gas anesthesia, sevoflurane and intravenous anesthesia and propofol have been widely used during the CABG. To further understand the underlying mechanisms of these anesthetics on the gene level, the present study conducted pathway-related module analysis based on a co-expression network. This was performed in order to identify significant pathways in coronary artery disease patients who had undergone off-pump CABG surgery before and after applying sevoflurane or propofol. A total of 269 and 129 differentially expressed genes were obtained in the sevoflurane and propofol groups, respectively. In total, eight and seven pathways $(\mathrm{P}<0.05)$ in the sevoflurane and propofol groups were separately obtained via Kyoto Encyclopedia of Genes and Genome pathway analysis. Finally, eight and seven pathway-related modules in the sevoflurane and propofol groups were obtained, respectively. Furthermore, the mean degree of complement and coagulation cascades pathway-related module in both of the groups was the highest. It was predicted that during the $\mathrm{CABG}$, the anesthetics might activate the complement and coagulation systems in order to possess some cardioprotective properties.
\end{abstract}

Correspondence to: Mr. Feng Qi, Department of Anesthesiology, Qilu Hospital of Shandong University, 107 Wenhuaxi Road, Jinan, Shandong 250012, P.R. China

E-mail: aifengqi2015@sina.com

Key words: coronary artery disease, sevoflurane, propofol, pathway-related modules, co-expression network

\section{Introduction}

Cardiovascular diseases (CVDs) account for $>17$ million cases of mortality globally and annually (30\% of all mortality), $80 \%$ of which occur in low- and middle-income countries, and this figure is expected to grow to 23.6 million by 2030 (1). Coronary artery disease (CAD) is the largest contributor to CVDs (2). Off-pump coronary artery bypass graft (CABG) surgery emerged in recent years as a means to avoid the sequelae of extracorporeal circulation such as whole-body inflammatory response, coagulation disorders, and multiple organ dysfunction (3). At present, gas anesthesia-sevoflurane and intravenous anesthesia-propofol have been widely used during the CABG (4).

Propofol and sevoflurane both possess certain, although different, cardioprotective properties. Sevoflurane appeared to be superior to propofol in patients with little or no ischemic heart disease, including CABG surgery without severe preoperative ischemia (5). However, propofol appeared superior in patients with cardiovascular instability, severe ischemia or acute/urgent surgery (6). Over the past decades, numerous experimental strategies (association studies, genome-wide linkage scan, proteomics and global microarray gene expression analysis amongst others and large efforts have been applied onto the studies $(7,8)$. Lucchinetti et al (9) performed direct comparisons between anesthetic gases and intravenous anesthetics in human hearts at the gene expression level. These results indicated that anesthetic-induced and constitutive gene regulatory control of myocardial substrate metabolism predicts postoperative cardiac function in patients undergoing off-pump CABG surgery. However, the underlying mechanisms of these anesthetics on the gene level remain unclear.

Previous studies have established a constructed gene co-expression network, which contained genes that exhibited similar expression patterns across different organisms $(10,11)$. It has also been demonstrated that functionally related genes were frequently co-expressed across organisms constituting conserved transcription modules (12). By constructing a co-expression network, the underlying regulatory relationships under different conditions may be estimated (13). In order to 
define the adjacency matrix, one makes use of an adjacency function, which transforms the co-expression similarities into connection strengths (14). The node dissimilarity measure is used as input of a clustering method to define network modules (clusters of nodes) (15). Furthermore, modules are groups of genes whose expression profiles are highly correlated across the samples (16). Network modules implement the hypothesis that a network can be divided into functional modules (17). In this case, significant interactions, such as key genes in significant pathways can be tested. Therefore, in the present study modules from the co-expression network based on genes enriched in significant pathways were identified, and these modules were defined as pathway-related modules.

The present study aimed to identify changed pathway-related modules in CAD patients undergoing CABG under sevoflurane or propofol anesthesia based on network topological centralities. In order to achieve this, recruitment and preprocessing of the gene expression profile was initially conducted, and differentially expressed genes (DEGs) in CAD patients were identified before and after applying sevoflurane or propofol, respectively. Next, pathway analysis of the DEGs was performed using the Kyoto Encyclopedia of Genes and Genomes database. A co-expression network was constructed by weighted gene co-expression network analysis (WGCNA), and pathway-related modules were mined. Finally, significant pathway-related modules were identified by conducting analysis on the topological centralities of the co-expression network, in order to further understand the underlying mechanisms of these anesthetics on the CAD patients during the CABG process according to systematically analyzing the pathway-related modules of the co-expression network.

\section{Materials and methods}

Data recruitment and preprocessing. The gene expression profile of E-GEOD-4386 was obtained from the ArrayExpress database (http://www.ebi.ac.uk/arrayexpress/). E-GEOD-4386 existed on the A-AFFY-44-Affymetrix GeneChip Human Genome U133 Plus 2.0 Platform. The data were obtained from patients that had undergone off-pump CABG surgery, and they were allocated either to receive the anesthetic gas sevoflurane or the intravenous anesthetic propofol. The samples were then divided into two groups: Baseline sevoflurane $(n=10)$-sevoflurane $(n=10)$ and baseline propofol $(n=10)$-propofol $(n=10)(9)$. Furthermore, the microarray data and annotation files were downloaded for further analysis.

Background-corrected signal intensities were determined using the Micro Array Suite 5.0 (MAS 5.0) software (Affymetrix, Inc., Santa Clara, CA, USA) (18). The normalization of datasets obtained from the ArrayExpress database was performed using a robust multichip average method (19) and quantile based algorithm (20). Meanwhile, the gene expression value was transformed to a comparable level. Additionally, a gene-filter package was used to screen the data. Each probe was mapped to one gene, and the probe was discarded if it did not match any genes. Furthermore, the expression value averaged over probes was used as the gene expression value if the gene had multiple probes, and 20,102 genes were obtained overall.
Identification of DEGs. The linear model for microarray data (LIMMA) package, a core component of Bioconductor, is an R-based open-source software development project in statistical genomics (21). A core capability is the use of linear models to assess differential expression in the context of multifactor designed experiments. In the present study, for genes with scores greater than an adjustable threshold, empirical Bayes method that was implemented in the package (22) was used to identify DEGs in the sevoflurane and propofol groups, respectively. Furthermore, the false discovery rate was used to proofread the P-values. Values of [log Fold Change (FC)] $>2.0$ and $\mathrm{P}<0.01$ were selected as the cut-off criteria.

Functional enrichment analysis of DEGs. KEGG pathway database is a recognized and comprehensive database including all types of biochemical pathways (23). In the present study, the KEGG database was applied to investigate the enrichment analysis of the nodes in order to find the biochemical pathways of DEGs that were involved in patients that had undergone off-pump CABG surgery before and after applying sevoflurane or propofol. The Database for Annotation, Visualization and Integrated Discovery (DAVID) (24) was used to perform the KEGG pathway enrichment analysis with the $\mathrm{P}<0.05$ and gene count $>5$.

\section{Co-expression network analysis}

Identifying differential co-expression by WGCNA. Gene co-expression networks, which represent a major application of correlation network methodology, are instrumental for describing the pair-wise relationships among gene transcripts and facilitate the understanding of their function and identification of their key players $(25,26)$. WGCNA, as a statistical approach based on correlations, has been widely used to analyze transcriptional profiles, and has proved to be an informative approach for the functional annotation of uncharacterized genes (27). A coefficient of variation $(\mathrm{CV}=\mu / \sigma)$ filtering was applied to remove genes that were constitutively expressed, unexpressed or vary only modestly across experimental treatments or conditions (28). In this study, a CV cutoff value of 0.6 was selected to obtain co-expression interactions.

Co-expression network construction. Cytoscape provides an environment for the visualization and analysis of networks and associated annotations (29). The primary audience for Cytoscape is the biological community, and it supports a number of standard use cases for analyzing and visualizing biological data (30). In the present study, the co-expression network was constructed using Cytoscape version 3.1.0. Meanwhile, the expression values of each node were mapped to the co-expression network, where different colors represent the differences in the expression value of the nodes.

Pathway-related module mining and topological analysis. In the present study, pathway-related modules were extracted from a co-expression network in order to investigate significant genes and modules that played key roles in patients undergoing off-pump CABG surgery before and after applying sevoflurane or propofol. To achieve this, firstly, genes in each significant pathway of the two groups were explored and mapped into the co-expression network. Next, pathway genes in the network 
Table I. KEGG pathway of DEGs in the sevoflurane group.

\begin{tabular}{llrr}
\hline ID & \multicolumn{1}{c}{ Term } & Count & P-value \\
\hline hsa04621 & NOD-like receptor signaling pathway & 11 & $7.39 \times 10^{-8}$ \\
hsa04060 & Cytokine-cytokine receptor interaction & 17 & $7.92 \times 10^{-6}$ \\
hsa04610 & Complement and coagulation cascades & 9 & $2.06 \times 10^{-5}$ \\
hsa05219 & Bladder cancer & 5 & $5.68 \times 10^{-3}$ \\
hsa04010 & MAPK signaling pathway & 12 & $5.92 \times 10^{-3}$ \\
hsa04115 & p53 signaling pathway & 6 & $6.13 \times 10^{-3}$ \\
hsa04062 & Chemokine signaling pathway & 9 & $1.50 \times 10^{-2}$ \\
hsa04630 & Jak-STAT signaling pathway & 8 & $1.72 \times 10^{-2}$ \\
\end{tabular}

KEGG, Kyoto Encyclopedia of Genes and Genomes; DEG, differentially expressed genes; NOD, nucleotide-binding oligomerization domain; MAPK, mitogen-activated protein kinase; STAT, Janus kinase/signal transducers and activators of transcription.

Table II. KEGG pathway of DEGs in the propofol group.

\begin{tabular}{llrr}
\hline ID & \multicolumn{1}{c}{ Term } & Count & P-value \\
\hline hsa04621 & NOD-like receptor signaling pathway & 7 & $4.68 \times 10^{-5}$ \\
hsa04060 & Cytokine-cytokine receptor interaction & 11 & $4.14 \times 10^{-4}$ \\
hsa04010 & MAPK signaling pathway & 10 & $2.00 \times 10^{-3}$ \\
hsa04115 & p53 signaling pathway & 5 & $5.98 \times 10^{-3}$ \\
hsa05219 & Bladder cancer & 4 & $1.02 \times 10^{-2}$ \\
hsa05120 & Epithelial cell signaling in Helicobacter pylori infection & 4 & $3.67 \times 10^{-2}$ \\
hsa04610 & Complement and coagulation cascades & 4 & $3.80 \times 10^{-2}$
\end{tabular}

NOD, nucleotide-binding oligomerization domain; MAPK, mitogen-activated protein kinase.

and their adjacent genes were captured to form a sub-network, which were also called pathway-related modules. Finally, module topological analysis (the mean degree centrality of genes in the corresponding module) was conducted to evaluate significant pathway-related modules.

\section{Results}

Identifying DEGs. After having preprocessed the profile, the empirical Bayes method (F test) that was implemented in the LIMMA package was used to identify DEGs in the sevoflurane and propofol group. Under this condition, when the threshold values of $[\log (\mathrm{FC})]>2.0$ and $\mathrm{P}<0.01$ were set, a total of 269 DEGs were obtained in the sevoflurane group and a total of 129 DEGs in the propofol group.

KEGG pathway analysis of the DEGs. Based on human genomes, DAVID for KEGG pathway enrichment analysis was performed to further investigate the biological functions of the DEGs. When the threshold of P-value was set to 0.05, eight significant pathways in the sevoflurane (Table I) and seven in the propofol (Table II) groups were obtained.

It was evident that nucleotide-binding oligomerization domain (NOD)-like receptor signaling pathway, cytokine-cytokine receptor interaction, complement and coagulation cascades, mitogen-activated protein kinase and p53 signaling pathways were enriched in both groups. While the chemokine and Janus kinase/signal transducers and activators of transcription signaling pathways were only enriched in the sevoflurane group, epithelial cell signaling in Helicobacter pylori infection was only enriched in the propofol group.

Co-expression network construction and topological analysis. After having identified the DEGs, co-expression analysis was conducted on these 269 DEGs in the sevoflurane group and 129 DEGs in the propofol group using the WGCNA method. By setting a threshold CV cutoff value of 0.6, 813 (180 DEGs) and 1,216 (119 DEGs) co-expression interactions were obtained in two groups. Furthermore, two co-expression networks were obtained via a conducting network with the co-expression interactions in Cytoscape Version 3.1.0. Additionally, as we mapped the expression values of each node to the co-expression network separately, two networks with expression values were obtained (Figs. 1 and 2). It was evident that all of the pathway genes were in green $([\log (\mathrm{FC})]>2.0)$, which meant that these genes were all upregulated.

Pathway-related module mining and topological analysis. After having separately investigated genes in each significant pathway of the two groups, and having mapped them into the co-expression network, eight and seven pathway-related 


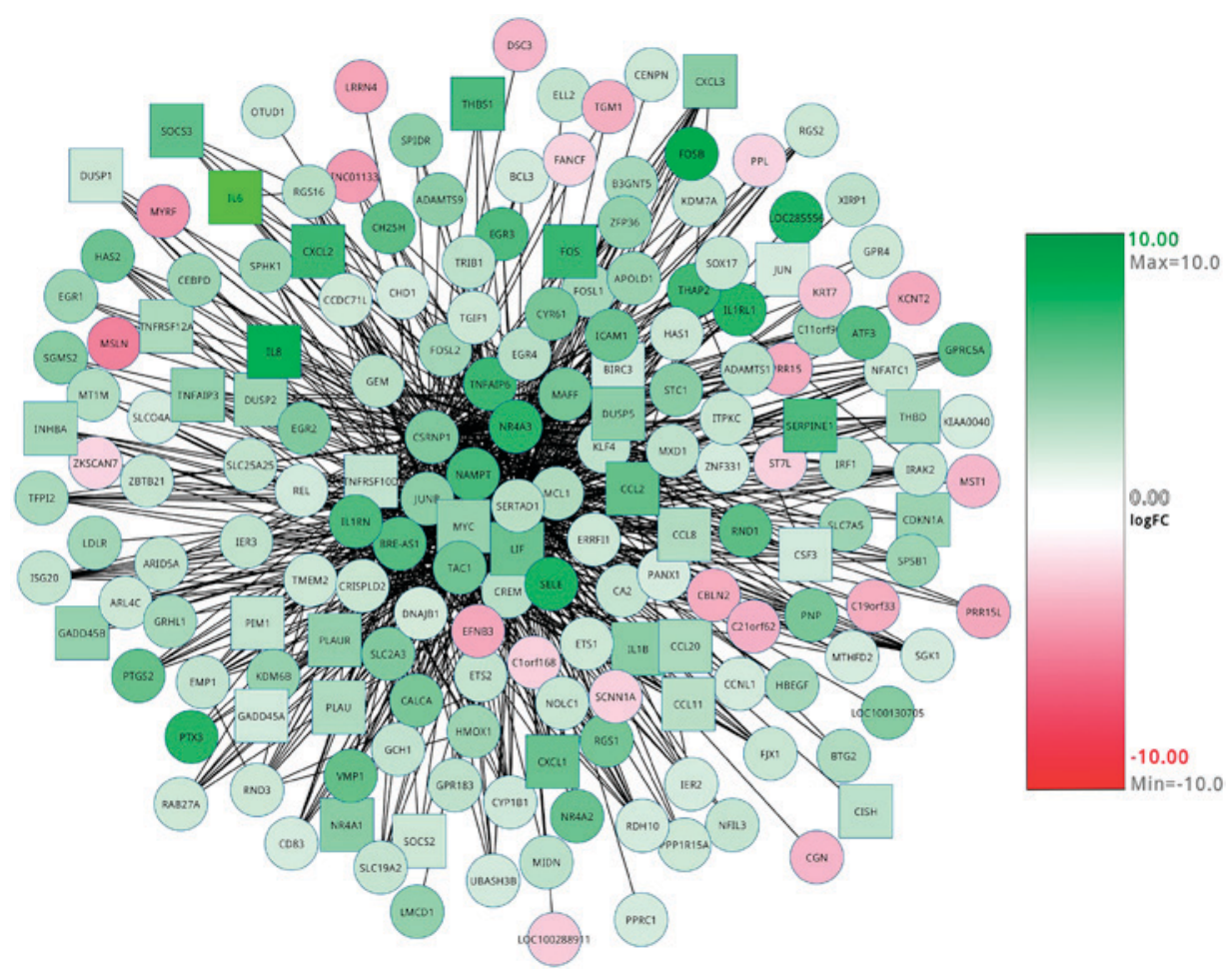

Figure 1. Co-expression network of DEGs in the sevoflurane group. The color represented the expression value of the genes, where dark red represented the minimum $(\log \mathrm{FC}=-10)$ and dark green the maximum $(\log \mathrm{FC}=10)$. The round nodes only consisted of DEGs whereas the square nodes consisted of $\mathrm{DEGs}$ and also other genes present in the pathways $(\mathrm{P}<0.05)$. DEG, differentially expressed genes; FC, fold change.

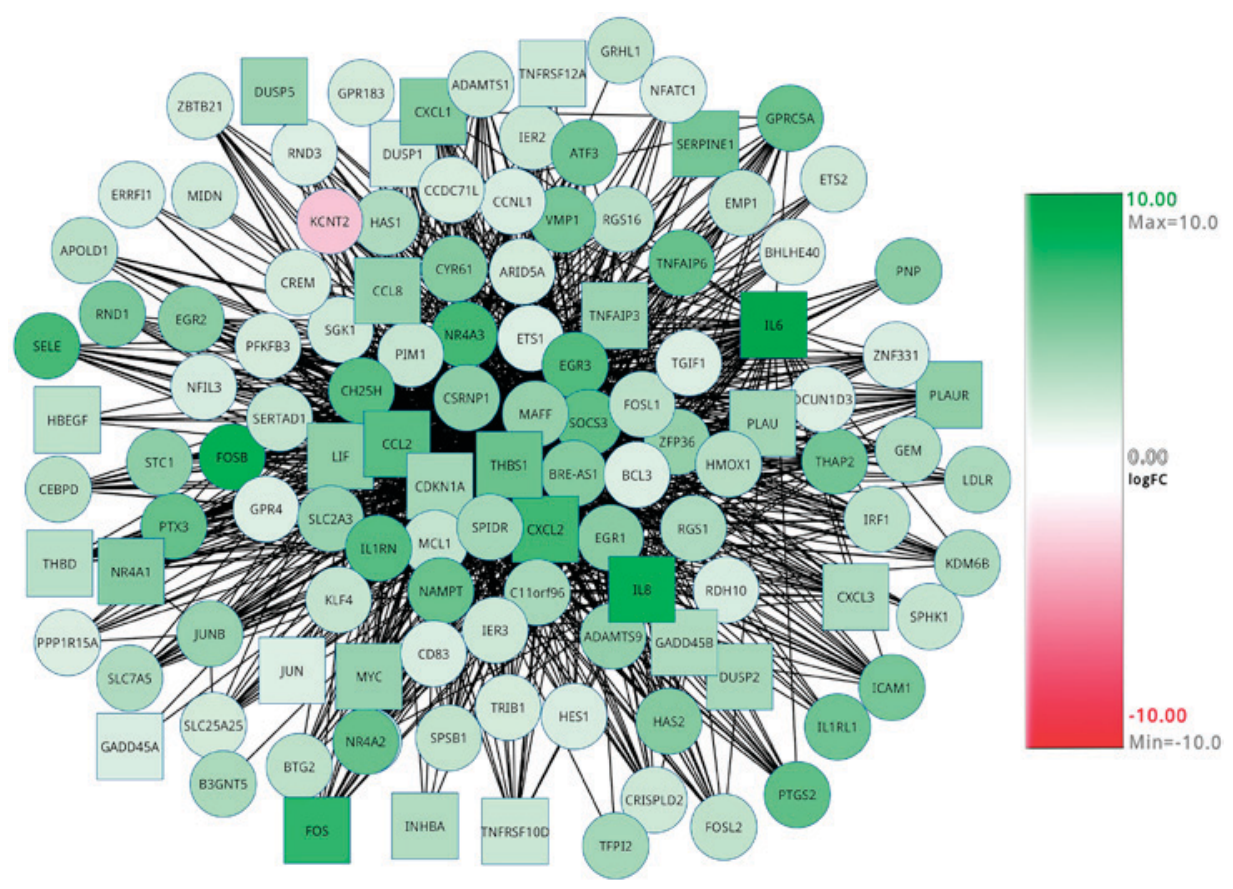

Figure 2. Co-expression network of DEGs in the propofol group. The color represented the expression value of the genes, where dark red represented the minimum $(\log F C=-10)$ and dark green the maximum $(\log F C=10)$. The round nodes only consisted of DEGs whereas the square nodes consisted of DEGs and also other genes present in the pathways $(\mathrm{P}<0.05)$. DEG, differentially expressed genes; FC, fold change.

modules were obtained in the sevoflurane group (Figs. 3 and 4) and in the propofol group (Figs. 5 and 6), respectively. To further investigate the biological functions of these modules, degree centrality analysis was performed, the details of which are shown in Table III (sevoflurane group) and Table IV (propofol group). The mean degree of modules of complement and coagulation cascades related, p53 signaling related, NOD-like receptor signaling related and cytokine-cytokine receptor interaction related were $>20$ in both of the groups. Moreover, the complement and coagulation cascades pathway-related 
A

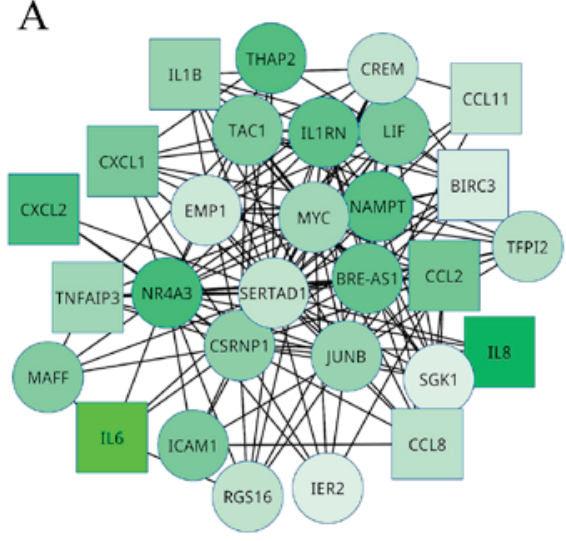

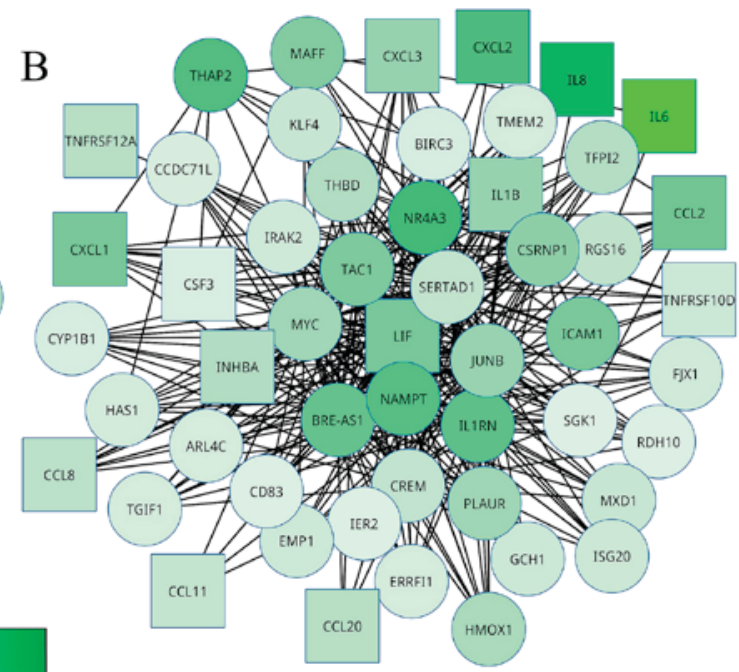

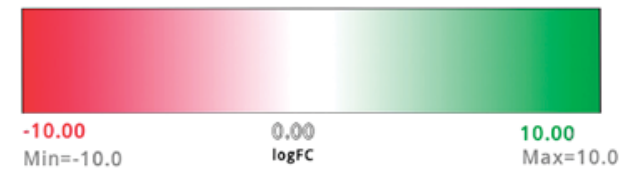

C

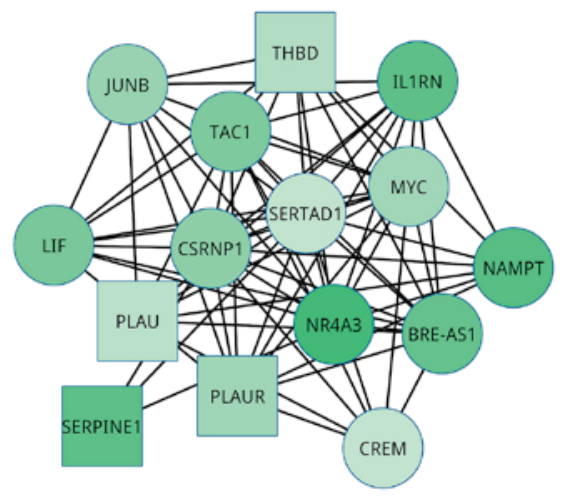

$\operatorname{Max}=10.0$

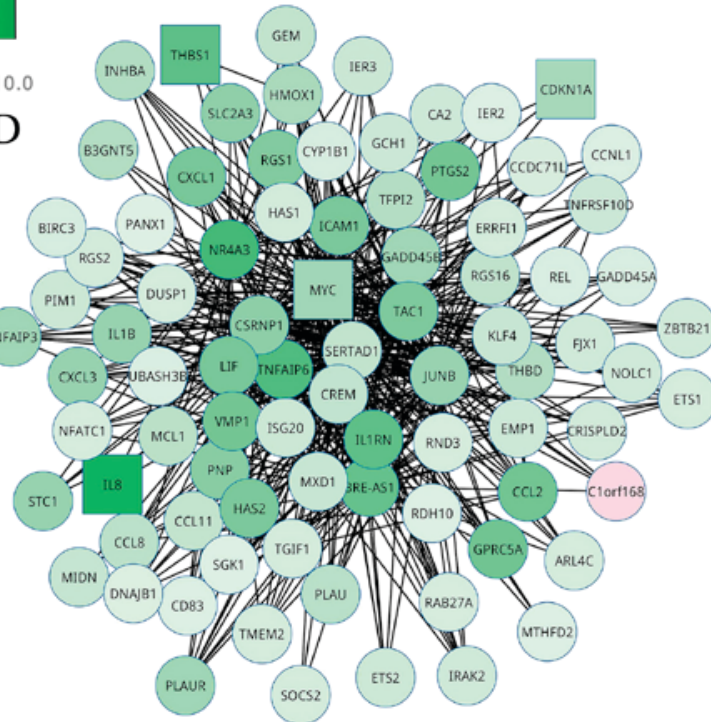

Figure 3. Pathway-related modules in the sevoflurane group. (A-D) Respectively represent the pathway-related modules of pathways 1-4 in the sevoflurane group. The color represented the expression value of the genes, where dark red represented the minimum (logFC $=-10)$ and dark green the maximum $(\log \mathrm{FC}=10)$. The square nodes represented pathway genes. FC, fold change.

module revealed the highest mean degree in both groups, which in the sevoflurane group had a mean degree of 56.67, and in the propofol group a mean degree of 71.88 . However, the chemokine signaling pathway-related module only existed in the sevoflurane group with a mean degree of 36.92 , and epithelial cell signaling in $H$. pylori infection pathway-related module only existed in the propofol group with a mean degree of 35.98 .

By conducting analysis on the frequency of genes contained in the pathway-related modules, it was identified that there were several genes that presented in more than one module in both of the groups, as presented in Table V (sevoflurane group) and Table VI (propofol group). Furthermore, it was evident that in the sevoflurane group, genes IL8, CXCL2, CCL2, IL6, IL1B, CXCL1, CCL11 and MYC had a frequency $>3$ and in the propofol group, genes IL8 and CXCL1 had a frequency $>3$. In addition, genes IL8, CXCL2, CCL2, CXCL1 and CCL11 were all enriched in the chemokine signaling pathway-related module of the sevoflurane group, and genes IL8, CXCL1 were all enriched in epithelial cell signaling in
H. pylori infection pathway-related module of the propofol group.

\section{Discussion}

In the present study, an analysis on the gene profiles of patients who had undergone off-pump CABG surgery before and after applying sevoflurane or propofol was conducted based on a pathway-related module associated co-expression network. Since the co-expression network that separately mapped all of the genes present in the significant pathways was analyzed, it was shown that the mean degree of several modules (complement and coagulation cascades pathway-related module, p53 signaling pathway-related module, NOD-like receptor signaling pathway-related module and cytokine-cytokine receptor interaction pathway-related module) were $>20$ in both of the groups, and the mean degree of complement and coagulation cascades pathway-related module in both of the groups were the highest. However, a chemokine signaling pathway-related module only existed in the sevoflurane group with a mean degree of 36.92 , and 


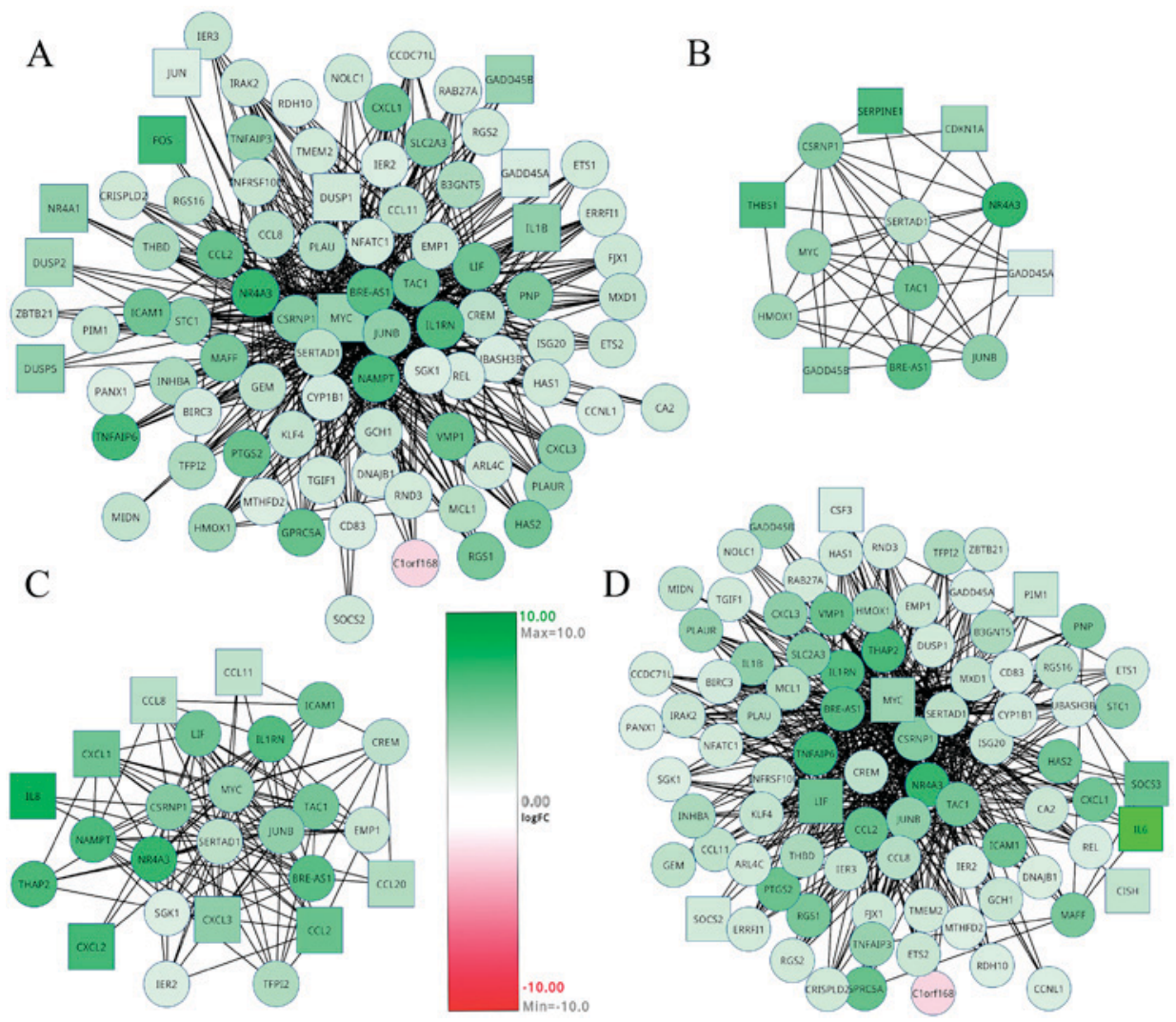

Figure 4. Pathway-related modules in the sevoflurane group. (A-D) Respectively represent the pathway-related modules of pathway 5-8 in the sevoflurane group. The color represented the expression value of the genes, where dark red represented the minimum $(\log \mathrm{FC}=-10)$ and dark green the maximum $(\log \mathrm{FC}=10)$. The square nodes represented the pathway genes. FC, fold change.
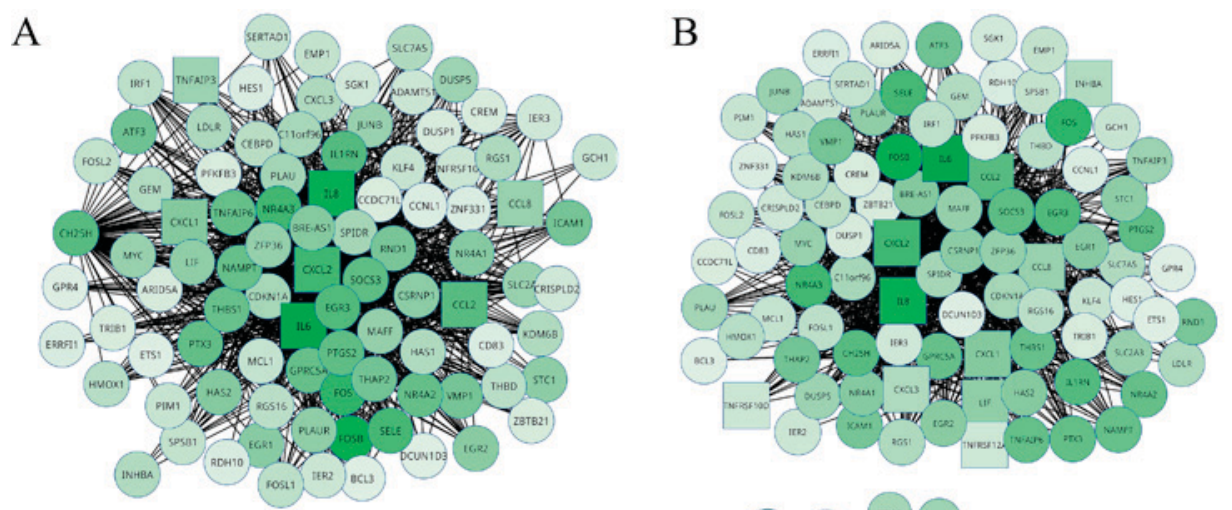

$\mathrm{C}$

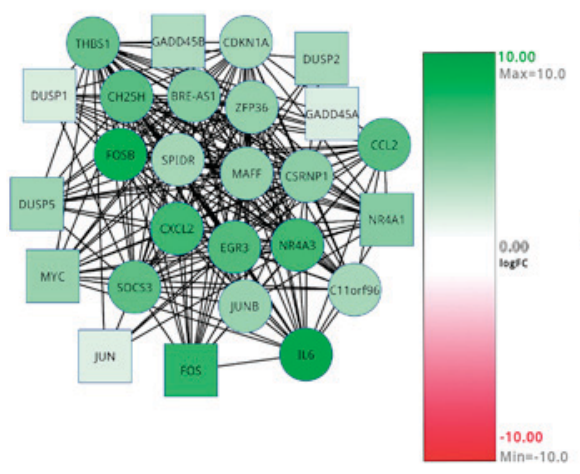

D

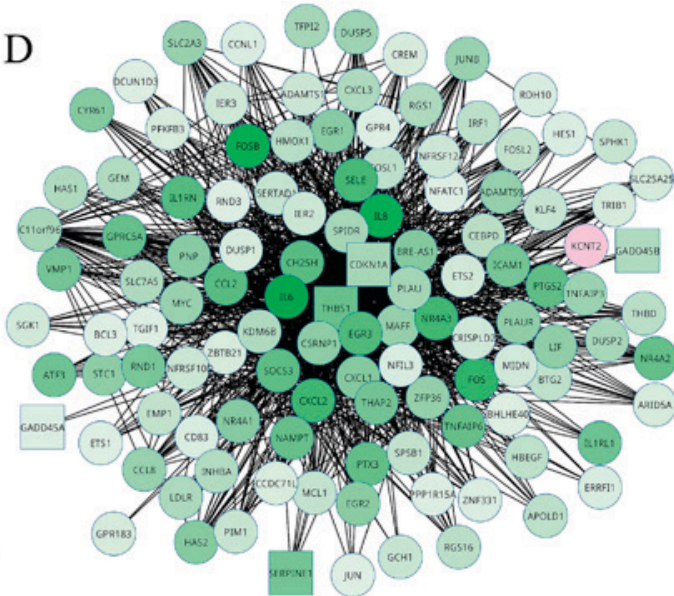

Figure 5. Pathway-related modules in the propofol group. (A-D) Respectively represent the pathway-related modules of pathway 1-4 in the propofol group. The color represented the expression value of the genes, where dark red represented the minimum $(\log \mathrm{FC}=-10)$ and dark green the maximum $(\log \mathrm{FC}=10)$. The square nodes represented pathway genes. FC, fold change. 

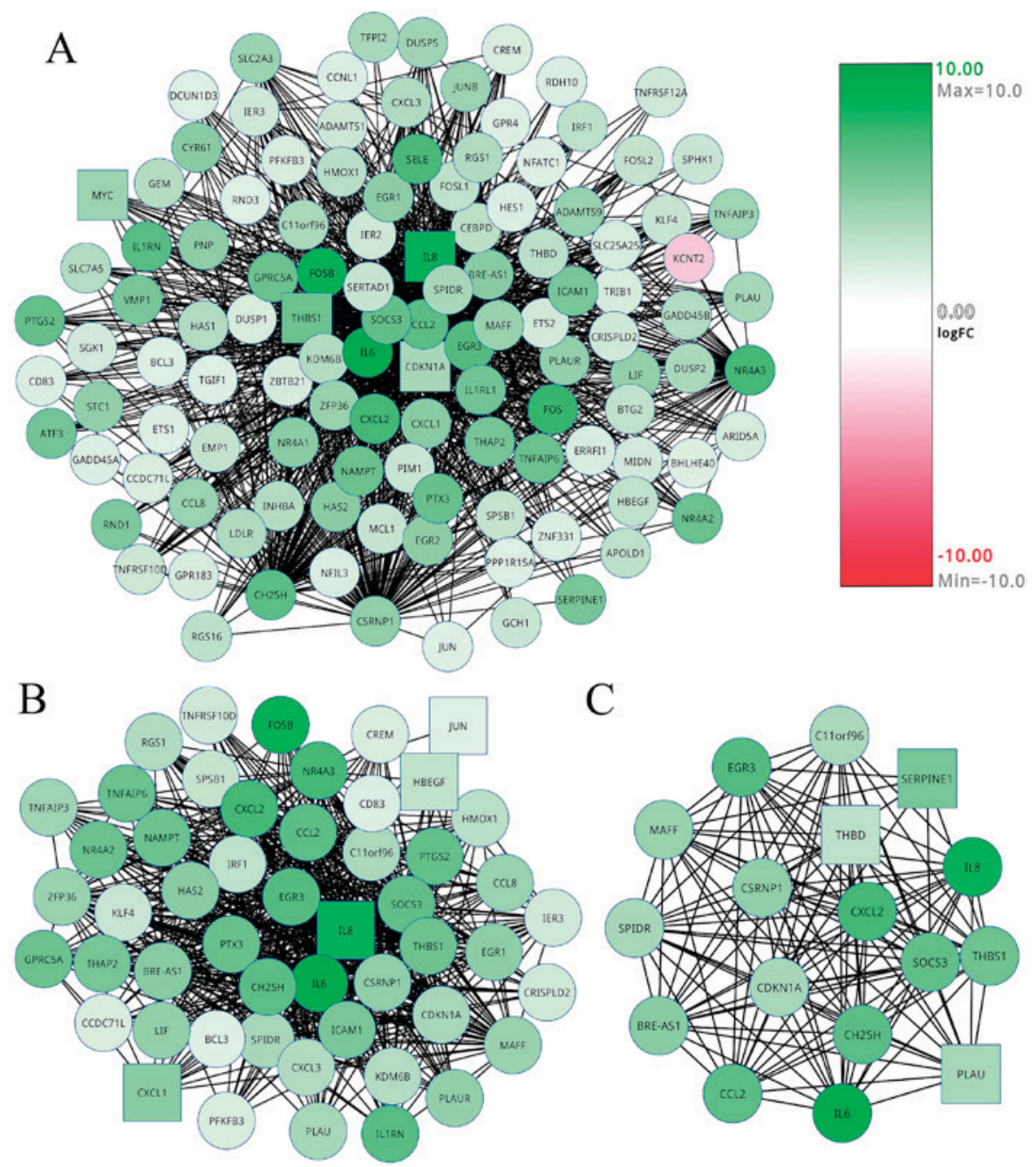

Figure 6. Pathway-related modules in the propofol group. (A-C) Respectively represents the pathway-related modules of pathway 5-7 in the propofol group. The color represented the expression value of the genes, where dark red represented the minimum $(\log F C=-10)$ and dark green the maximum $(\log F C=10)$. The square nodes represented pathway genes. FC, fold change.

epithelial cell signaling in $H$. pylori infection pathway-related module only existed in the propofol group with a mean degree of 35.98 .

The complement and coagulation systems were described as separate cascades and as descendants of a common ancestral pathway. Both proteolytic cascades were composed of serine proteases with common structural characteristics, including highly conserved catalytic sites of histidine, aspartate and serine $(31,32)$. Furthermore, both systems belonged to a complex inflammatory network (33) and exhibited some similar characteristics with regard to the specialized functions of their inhibitors and activators (34). It was indicated that cardiac surgery with cardiopulmonary bypass gives rise to a systemic inflammatory reaction, caused by the extracorporeal circuit and surgical trauma (35), which generated activation of the complement, fibrinolytic, kallikrein and coagulation cascades, activation of leukocytes and endothelial cells with expression of adhesion molecules and the release of inflammatory mediators, such as cytokines (36). Pathways of cytokine-cytokine receptor interaction were at the top of an enriched pathway list in an CAD gene KEGG pathway analysis (37).

Cytokines represent a diverse group of molecules that transmit intercellular signals. These signals may be paracrine or autocrine. Both of these situations could occur simultaneously (38). In addition, injured endothelial cells produce cytokines (including interleukin) that stimulate the expression of adhesion proteins selectins and cell adhesion molecules including vascular cell adhesion and intercellular adhesion molecules on the endothelial surface (39). It had been concluded that among apparently healthy women and men, elevated levels of IL8 are associated with an increased risk of CAD (40). There has also been increasing recognition that in various pathological conditions, CB1 receptor activation by endocannabinoids may promote activation of signaling pathways promoting cell death (41).

The chemokines are a family of low-molecular-weight proteins involved in leukocyte activation and migration (42). Significant advances have been made in understanding the role of chemokines and their receptors in cardiovascular diseases (43) tumor growth as well as metastasis (44) and inflammatory diseases (45). It has been indicated that the molecular mechanisms responsible for monocyte accumulation in plaque of atherosclerosis are likely to include chemokines and their receptors, as these molecules were major regulators of specific leukocyte trafficking (46). In the present study, the chemokine signaling pathway-related module only existed 
Table III. Mean degree centrality of pathway-related modules in the sevoflurane group.

\begin{tabular}{lr}
\hline Pathway-term & Degree \\
\hline Complement and coagulation cascades & 56.67 \\
p53 signaling pathway & 55.69 \\
Chemokine signaling pathway & 36.92 \\
NOD-like receptor signaling pathway & 33.31 \\
Cytokine-cytokine receptor interaction & 22.29 \\
MAPK signaling pathway & 15.67 \\
Bladder cancer & 15.64 \\
Jak-STAT signaling pathway & 15.52 \\
\hline NOD, nucleotide-binding oligomerization & domain; \\
mitogen-activated protein kinase; STAT, Janus kinase/signal trans- \\
ducers and activators of transcription.
\end{tabular}

Table IV. Mean degree centrality of pathway-related modules in the propofol group.

\begin{tabular}{lc}
\hline Pathway Term & Degree \\
\hline Complement and coagulation cascades & 71.88 \\
MAPK signaling pathway & 53.46 \\
Epithelial cell signaling in Helicobacter & 35.98 \\
pylori infection & \\
NOD-like receptor signaling pathway & 25.01 \\
Cytokine-cytokine receptor interaction & 24.79 \\
p53 signaling pathway & 20.77 \\
Bladder cancer & 20.76 \\
\hline
\end{tabular}

MAPK, mitogen-activated protein kinase; NOD, nucleotide-binding oligomerization domain.

in the sevoflurane group, which meant that this anesthetic might give the patients with more protection in hematopoiesis, angiogenesis, metastasis and tumor rejection or inflammatory diseases. However, it has also been reported that epithelial cell signaling in $H$. pylori infection was mainly associated with peptic ulceration, chronic gastritis and more rarely with gastric adenocarcinoma (47).

In conclusion, in the present study, complement and coagulation cascade related modules were successfully identified to be significant in both groups of sevoflurane and propofol, which meant that during the CABG, these anesthetics might activate the complement and coagulation systems so as to exert some cardioprotective properties. While the chemokine signaling pathway-related module only existed in the sevoflurane group, which meant that this anesthetic might provide the patients with more protection in hematopoiesis, angiogenesis, metastasis and tumor rejection or inflammatory diseases.

However, there remained certain limitations in the present study that must be taken into account. First of all, the sample size was not large enough. Additionally, an experimental verification analysis should be conducted in order to verify the
Table V. Frequency of genes that are in the pathway-related modulus of the sevoflurane group.

\begin{tabular}{|c|c|}
\hline Symbol & No \\
\hline IL8 & 4 \\
\hline CXCL2 & 3 \\
\hline CCL2 & 3 \\
\hline IL6 & 3 \\
\hline IL1B & 3 \\
\hline CXCL1 & 3 \\
\hline CCL11 & 3 \\
\hline MYC & 3 \\
\hline CCL8 & 2 \\
\hline CXCL3 & 2 \\
\hline CCL20 & 2 \\
\hline CSF3 & 2 \\
\hline LIF & 2 \\
\hline SERPINE1 & 2 \\
\hline CDKN1A & 2 \\
\hline THBS1 & 2 \\
\hline GADD45A & 2 \\
\hline GADD45B & 2 \\
\hline BIRC3 & 1 \\
\hline TNFAIP3 & 1 \\
\hline INHBA & 1 \\
\hline TNFRSF12A & 1 \\
\hline TNFRSF10D & 1 \\
\hline PLAUR & 1 \\
\hline SOCS2 & 1 \\
\hline CISH & 1 \\
\hline THBD & 1 \\
\hline PLAU & 1 \\
\hline DUSP1 & 1 \\
\hline NR4A1 & 1 \\
\hline FOS & 1 \\
\hline DUSP2 & 1 \\
\hline JUN & 1 \\
\hline DUSP5 & 1 \\
\hline CDKN1A & 1 \\
\hline CXCL2 & 1 \\
\hline SOCS3 & 1 \\
\hline PIM1 & 1 \\
\hline
\end{tabular}

results obtained by the bioinformatics method used in the present study. Although disadvantages existed, it is believed that this method and the results offered investigators valuable resources for better understanding the underlying mechanisms of sevoflurane and propofol of the CAD patients who undergo CABG on the pathway level.

\section{Acknowledgements}

The present study was supported by the Department of Anesthesiology of Qilu Hospital of Shandong University, 
Table VI. Frequency of genes that are in the pathway-related modulus of the propofol group.

\begin{tabular}{|c|c|}
\hline Symbol & No. \\
\hline IL8 & 4 \\
\hline CXCL1 & 3 \\
\hline IL5 & 2 \\
\hline CXCL2 & 2 \\
\hline CCL2 & 2 \\
\hline CCL8 & 2 \\
\hline GADD45A & 2 \\
\hline GADD45B & 2 \\
\hline MYC & 2 \\
\hline DUSP2 & 2 \\
\hline JUN & 2 \\
\hline SERPINE1 & 2 \\
\hline THBS1 & 2 \\
\hline CDKN1A & 2 \\
\hline TNFAIP3 & 1 \\
\hline LIF & 1 \\
\hline TNFRSF10D & 1 \\
\hline INHBA & 1 \\
\hline TNFRSF12A & 1 \\
\hline CXCL3 & 1 \\
\hline DUSP5 & 1 \\
\hline DUSP1 & 1 \\
\hline NR4A1 & 1 \\
\hline FOS & 1 \\
\hline HBEGF & 1 \\
\hline THBD & 1 \\
\hline PLAU & 1 \\
\hline PLAUR & 1 \\
\hline
\end{tabular}

the Departments of Anesthesiology and Neurosurgery of the Affiliated Hospital of Binzhou Medical College. The authors would like to thank all the members of the research group. Meanwhile, the authors are grateful to the Ji'nan Evidence Based Medicine Science-Technology Center, who provided technical support during the data processing and analysis.

\section{References}

1. Mendis S, Puska P and Norrving B: Global atlas on cardiovascular disease prevention and control, 2011.

2. Wong ND: Epidemiological studies of CHD and the evolution of preventive cardiology. Nat Rev Cardiol 11: 276-289, 2014.

3. Scott NB, Turfrey DJ, Ray DA, Nzewi O, Sutcliffe NP, Lal AB, Norrie J, Nagels WJ and Ramayya GP: A prospective randomized study of the potential benefits of thoracic epidural anesthesia and analgesia in patients undergoing coronary artery bypass grafting. Anesth Analg 93: 528-535, 2001.

4. Heindl B, Reichle FM, Zahler S, Conzen PF and Becker BF: Sevoflurane and isoflurane protect the reperfused guinea pig heart by reducing postischemic adhesion of polymorphonuclear neutrophils. Anesthesiology 91: 521-530, 1999.

5. Jakobsen CJ, Berg H, Hindsholm KB, Faddy N and Sloth E: The influence of propofol versus sevoflurane anesthesia on outcome in 10,535 cardiac surgical procedures. J Cardiothorac Vasc Anesth 21: 664-671, 2007.
6. Jakobsen CJ, Berg H, Hindsholm KB, Faddy N and Sloth E: The influence of propofol versus sevoflurane anesthesia on outcome in 10,535 cardiac surgical procedures. J Cardiothorac Vasc Anesth 21: 664-671, 2007.

7. Karwacki Z, Kowiański P, Moryś J, Dziewiatkowski J, Kaczmarek E and Suchorzewska J: Effect of sevoflurane on intracranial pressure and cardiovascular function in rabbits with experimental intracerebral haematoma. Med Sci Monit 7: 212-217, 2001.

8. Conzen PF, Fischer S, Detter C and Peter K: Sevoflurane provides greater protection of the myocardium than propofol in patients undergoing off-pump coronary artery bypass surgery. Anesthesiology 99: 826-833, 2003.

9. Lucchinetti E, Hofer C, Bestmann L, Hersberger M, Feng J, Zhu M, Furrer L, Schaub MC, Tavakoli R, Genoni M, et al: Gene regulatory control of myocardial energy metabolism predicts postoperative cardiac function in patients undergoing off-pump coronary artery bypass graft surgery: Inhalational versus intravenous anesthetics. Anesthesiology 106: 444-457, 2007.

10. Stuart JM, Segal E, Koller D and Kim SK: A gene-coexpression network for global discovery of conserved genetic modules. Science 302: 249-255, 2003.

11. Bergmann S, Ihmels J and Barkai N: Similarities and differences in genome-wide expression data of six organisms. PLoS Biol 2: E9, 2004.

12. Choi JK, Yu U, Yoo OJ and Kim S: Differential coexpression analysis using microarray data and its application to human cancer. Bioinformatics 21: 4348-4355, 2005.

13. Basso K, Margolin AA, Stolovitzky G, Klein U, Dalla-Favera R and Califano A: Reverse engineering of regulatory networks in human B cells. Nat Genet 37: 382-390, 2005.

14. Zhang B and Horvath S: A general framework for weighted gene co-expression network analysis. Stat Appl Genet Mol Biol 4: Article 17, 2005.

15. Mumford JA, Horvath S, Oldham MC, Langfelder P, Geschwind DH and Poldrack RA: Detecting network modules in fMRI time series: A weighted network analysis approach. Neuroimage 52: 1465-1476, 2010.

16. Ravasz E, Somera AL, Mongru DA, Oltvai ZN and Barabasi AL: Hierarchical organization of modularity in metabolic networks. Science 297: 1551-1555, 2002.

17. Davidson EH, McClay DR and Hood L: Regulatory gene networks and the properties of the developmental process. Proc Natl Acad Sci USA 100: 1475-1480, 2003.

18. Pepper SD, Saunders EK, Edwards LE, Wilson CL and Miller CJ: The utility of MAS5 expression summary and detection call algorithms. BMC Bioinformatics 8: 273, 2007.

19. Ma L, Robinson LN and Towle HC: ChREBP* Mlx is the principal mediator of glucose-induced gene expression in the liver. J Biol Chem 281: 28721-28730, 2006.

20. Rifai N and Ridker PM: Proposed cardiovascular risk assessment algorithm using high-sensitivity $\mathrm{C}$-reactive protein and lipid screening. Clin Chem 47: 28-30, 2001.

21. Gentleman RC, Carey VJ, Bates DM, Bolstad B, Dettling M, Dudoit S, Ellis B, Gautier L, Ge Y, Gentry J, et al: Bioconductor: Open software development for computational biology and bioinformatics. Genome Biol 5: R80, 2004.

22. Ritchie ME, Silver J, Oshlack A, Holmes M, Diyagama D, Holloway A and Smyth GK: A comparison of background correction methods for two-colour microarrays. Bioinformatics 23: 2700-2707, 2007.

23. Kanehisa M and Goto S: KEGG: Kyoto encyclopedia of genes and genomes. Nucleic Acids Res 28: 27-30, 2000.

24. Huang DW, Sherman BT, Tan Q, Collins JR, Alvord G, Roayaei J, Stephens R, Baseler MW, Lane HC and Lempicki RA: The DAVID gene functional classification tool: A novel biological module-centric algorithm to functionally analyze large gene lists. Genome Biol 8: R183, 2007.

25. Carey VJ, Gentry J, Whalen E and Gentleman R: Network structures and algorithms in Bioconductor. Bioinformatics 21: 135-136, 2005

26. Cokus S, Rose S, Haynor D, Grønbech-Jensen N and Pellegrini M: Modelling the network of cell cycle transcription factors in the yeast Saccharomyces cerevisiae. BMC Bioinformatics 7: 381, 2006.

27. Iancu OD, Darakjian P, Walter NA, Malmanger B, Oberbeck D, BelknapJ,McWeeney SandHitzemannR:Genetic diversityand striatalgenenetworks: Focus on the heterogeneousstock-collaborative cross (HS-CC) mouse. Bmc Genomics 11: 585, 2010. 
28. Childs KL, Davidson RM and Buell CR: Gene coexpression network analysis as a source of functional annotation for rice genes. PLoS One 6: e22196, 2011.

29. Shannon P, Markiel A, Ozier O, Baliga NS, Wang JT, Ramage D, Amin N, Schwikowski B and Ideker T: Cytoscape: A software environment for integrated models of biomolecular interaction networks. Genome Res 13: 2498-2504, 2003.

30. Cline MS, Smoot M, Cerami E, Kuchinsky A, Landys N, Workman C, Christmas R, Avila-Campilo I, Creech M, Gross B, et al: Integration of biological networks and gene expression data using Cytoscape. Nat Protoc 2: 2366-2382, 2007.

31. Krem MM and Di Cera E: Evolution of enzyme cascades from embryonic development to blood coagulation. Trends Biochem Sci 27: 67-74, 2002.

32. Esmon CT: The impact of the inflammatory response on coagulation. Thromb Res 114: 321-327, 2004.

33. Rittirsch D, Flierl MA and Ward PA: Harmful molecular mechanisms in sepsis. Nat Rev Immunol 8: 776-787, 2008.

34. Amara U: Molecular intercommunication between the complement and coagulation systems. J Immunology 185: 5628-5636, 2010.

35. Levy JH and Tanaka KA: Inflammatory response to cardiopulmonary bypass. Ann Thorac Surg 75: S715-S720, 2003.

36. Wehlin L, Vedin J, Vaage J and Lundahl J: Activation of complement and leukocyte receptors during on-and off pump coronary artery bypass surgery. Eur J Cardiothorac Surg 25: 35-42, 2004.

37. Liu H, Liu W, Liao Y, Cheng L, Liu Q, Ren X, Shi L, Tu X, Wang QK and Guo AY: CADgene: A comprehensive database for coronary artery disease genes. Nucleic Acids Res 39 (Database issue): D991-D996, 2011.

38. Leonard WJ and Lin JX: Cytokine receptor signaling pathways. J Allergy Clin Immunol 105: 877-888, 2000.
39. Auer J, Weber T, Berent R, Lassnig E, Lamm G and Eber B: Genetic polymorphisms in cytokine and adhesion molecule genes in coronary artery disease. Am J Pharmacogenomics 3: 317-328, 2003.

40. Boekholdt SM, Peters RJ, Hack CE, Day NE, Luben R, Bingham SA, Wareham NJ, Reitsma PH and Khaw KT: IL-8 plasma concentrations and the risk of future coronary artery disease in apparently healthy men and women: The EPIC-Norfolk prospective population study. Arterioscler Thromb Vasc Biol 24: 1503-1508, 2004.

41. Dalton GD, Bass CE, Van Horn CG and Howlett AC: Signal transduction via cannabinoid receptors. CNS Neurol Disord Drug Targets 8: 422-431, 2009.

42. Mellado M, Rodríguez-Frade JM, Mañes S and Martínez AC: Chemokine signaling and functional responses: The role of receptor dimerization and TK pathway activation. Annu Rev Immunol 19: 397-421, 2001.

43. Wang JM, Shen W and Su S: Chemokines and their role in cardiovascular diseases. Trends Cardiovasc Med 8: 169-174, 1998.

44. Rossi D and Zlotnik A: The biology of chemokines and their receptors. Annu Rev Immunol 18: 217-242, 2000.

45. Murdoch $\mathrm{C}$ and Finn A: Chemokine receptors and their role in inflammation and infectious diseases. Blood 95: 3032-3043, 2000.

46. Moatti D, Faure S, Fumeron F, Amara Mel-W, Seknadji P, McDermott DH, Debré P, Aumont MC, Murphy PM, de Prost D and Combadière $\mathrm{C}$ : Polymorphism in the fractalkine receptor CX3CR1 as a genetic risk factor for coronary artery disease. Blood 97: 1925-1928, 2001.

47. Crabtree JE and Naumann M: Epithelial Cell Signaling in Helicobacter pylori Infection. Curr Sig Transduc Ther 1: 53-65, 2006. 